\title{
EIGER: Electromagnetic Interactions GEneRalized
}

\author{
N. J. Champagne, R. M. Sharpe, J. W. Rockway
}

This article was submitted to Department of Defense High Performance Computing Modernization Program Users Group Conference 2001, Biloxi, MS., June 18-21, 2001

\section{June 13, 2001}

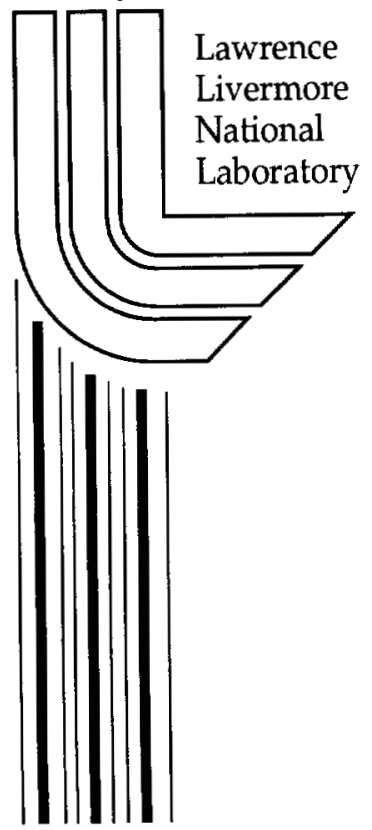




\section{DISCLAIMER}

This document was prepared as an account of work sponsored by an agency of the United States Government. Neither the United States Government nor the University of California nor any of their employees, makes any warranty, express or implied, or assumes any legal liability or responsibility for the accuracy, completeness, or usefulness of any information, apparatus, product, or process disclosed, or represents that its use would not infringe privately owned rights. Reference herein to any specific commercial product, process, or service by trade name, trademark, manufacturer, or otherwise, does not necessarily constitute or imply its endorsement, recommendation, or favoring by the United States Government or the University of California. The views and opinions of authors expressed herein do not necessarily state or reflect those of the United States Government or the University of California, and shall not be used for advertising or product endorsement purposes.

This is a preprint of a paper intended for publication in a journal or proceedings. Since changes may be made before publication, this preprint is made available with the understanding that it will not be cited or reproduced without the permission of the author.

This work was performed under the auspices of the United States Department of Energy by the University of California, Lawrence Livermore National Laboratory under contract No. W-7405-Eng-48.

This report has been reproduced directly from the best available copy.

Available electronically at http://www.doc.gov/bridge

Available for a processing fee to U.S. Department of Energy

And its contractors in paper from

U.S. Department of Energy

Office of Scientific and Technical Information

P.O. Box 62

Oak Ridge, TN 37831-0062

Telephone: (865) 576-8401

Facsimile: (865) 576-5728

E-mail: reports@adonis.osti.gov

Available for the sale to the public from

U.S. Department of Commerce

National Technical Information Service

5285 Port Royal Road

Springfield, VA 22161

Telephone: (800) 553-6847

Facsimile: (703) 605-6900

E-mail: orders@ntis.fedworld.gov

Online ordering: http://www.ntis.gov/ordering.htm

OR

Lawrence Livermore National Laboratory

Technical Information Department's Digital Library

http://www.llnl.gov/tid/Library.html 


\title{
EIGER: Electromagnetic Interactions GEneRalized
}

\author{
Nathan J. Champagne and Robert M. Sharpe \\ Lawrence Livermore National Laboratory \\ Livermore, CA 94550 \\ John W. Rockway \\ Space and Naval Warfare Systems Center \\ San Diego, CA 95152
}

\begin{abstract}
The EIGER (Electromagnetic Interactions Generalized) modeling suite is a joint development activity by the Lawrence Livermore National Lab, Sandia National Labs, the University of Houston, and the Navy (Space and Naval Warfare Systems Center - San Diego). The effort endeavors to bring the next generation of hybrid, higher-order, fullwave analysis methods into a single integrated framework. The tools are based upon frequency-domain solutions of Max well's equations to model scattering and radiation from complex 2D and 3D structures. The framework employs boundary element solutions of integral equation formulations and finite element solutions of the Helmholtz wave equation. A goal is to use higher-order representations to model both the geometry (using higher-order geometric elements) and numerical methods (using higher-order vector basis functions). In addition, a variety of advanced Green's functions and symmetry operators can be applied to efficiently treat geometries containing such features as layered material regions and periodic structures. Each of these methods can be brought to bear simultaneously, on different portions of a complex structure. HPC implementation issues were addressed during the design of the software architecture, so that the same package runs on platforms ranging from serial desktop workstations through advanced HPC architectures. Our current efforts on higher-order modeling and improved solver libraries will be highlighted.
\end{abstract}

\section{Introduction}

EIGER (Electromagnetic Interactions GEneRalized) is a single integrated software tool set that represents electromagnetic interactions in a full-wave, completely self-consistent manner. It is ideally suited to the computation of electromagnetic scattering and the radar cross section of complex bodies in the resonance regime and beyond. The application base for the EIGER code suite is as varied as the methods that are incorporated in the code. The tools have been used to electromagnetically model such diverse application areas as micro-electromechanical (MEMs) systems, extreme ultraviolet (EUV) lithography, broadband and array antenna systems, as well as computing the scattering and radar cross section (RCS) of complex bodies.

EIGER brings together a variety of spectral domain analysis methods. These include moment method solutions of integral equation formulations and finite element solutions of partial differential equations. As such, it is limited only by the constraints of available computational resources, but work on relieving even these constraints continues. The 
code suite was developed to run seamlessly on the full range of platforms from Intelbased PCs to Unix-based workstations to massively parallel systems. In fact, the same software suite runs on the state-of-the-art supercomputing platforms operated by the DOE, DoD, and intelligence community.

The extraordinary capabilities of EIGER are enabled by a number of approaches that are novel to the electromagnetics community. New software engineering methods, specifically, object-oriented design, are being used to implement abstractions of key components of spectral analysis methods so that the tools can be easily modified and extended to treat new classes of problems. The key components of the numerical analysis tool, and their roles, are: elements - to describe the geometry, basis functions - to interpolate the unknowns (e.g., fields) locally, and operators - to express the underlying physics formulations used to propagate the energy or enforce fundamental principals. These abstractions are discussed more fully in subsequent sections.

This design approach is in contrast to standard design procedures where entire codes are developed around a particular element type with a specific basis function for a single operator. Although such tools can be effectively used to model large classes of problems, it is often very difficult, if not intractable, to extend the tools beyond their initial design. Overcoming this limitation was one of the most compelling goals of this development. Moreover, the application base for EIGER grows as we cast a variety of analytical treatments (via Green's functions) into a form compatible with the numerical procedures in EIGER.

\section{Elements}

Elements are the basic building blocks that are used to describe a given geometry for numerical computation. These elements are typically the output of a commercial mesh generation package that has discretized a solid CAD model into pieces that are amenable to numerical computation. A pre-processor then reads these elements and assigns such features as material characteristics and excitation parameters to generate the actual input for the physics code.

The EIGER suite can treat both two and three-dimensional problems within its single integrated tool set. Initially, emphasis was placed on the requirements for modeling surface physics. Thus, for two-dimensional geometries, combinations of linear segments or "bar" elements are currently used to describe the surface problem. Likewise, for threedimensional geometries, both planar triangles and rectangles can be combined to render accurate surface models. In addition to the basic elements for modeling 3D surfaces, wire segments are also incorporated into EIGER.

A scheme to employ higher-order surface elements to more accurately resolve the geometry of curved objects, based on [3], is being implemented. These elements can be of arbitrary order, and will more accurately resolve the local variations of the geometry. Also, higher-order volume elements are being incorporated for treating inhomogeneous 
material regions along with solvers tuned for the resulting sparse systems that arise from a finite element approach.

\section{Basis Functions}

Basis functions are used to locally interpolate each unknown quantity. These unknowns may physically represent surface currents or fields for dynamic operators, or potentials for statics. Both constant and linear basis functions are currently available for each of the elements described above. The characteristic integrals (for integral equation solutions) that arise in many problems require that the potentials and their gradients due to source distributions that are represented by these basis functions must be efficiently computed. Extensive efforts have enabled most of these calculations to be cast into the same basic form, thus simplifying their integration into a general-purpose code.

In conjunction with the efforts to add higher-order elements, higher-order basis functions are being implemented, based on the formulations in [3], to enable a more efficient numerical solution of electrically large problems. In addition, a new formulation for singular basis functions, which will incorporate known edge conditions and other known local variations directly into the numerical procedure, is being developed.

\section{Operators}

An electromagnetic operator is a mathematical construct that relates the field at a point to the sources, which produced the field. Since previous efforts focused initially on incorporating surface physics, emphasis was placed on integral operators. These operators explicitly propagate a field between locations. A variety of integral operators have been incorporated into EIGER to treat both perfect electric conductors and homogeneous dielectrics (penetrable materials). Specifically, the following operators have been included: the Electric Field Integral Equation, the Magnetic Field Integral Equation, the Combined Field Integral Equation, and the PMCHW for general homogeneous lossy dielectrics.

The basic formulation for the integral operators has been generalized and simplified so that all of the above operators are generated by simply taking linear combinations of basic coupling or interaction operators. This powerful abstraction means that a variety of other boundary conditions (e.g., equivalent aperture formulations and alternative dielectric treatments) can easily be added to the code by simply choosing the appropriate set of coefficients.

\section{Green's Functions}

One of the most promising features of the EIGER development is the ability to directly incorporate a variety of Green's functions into the solution. In addition to the standard homogeneous free space type Green's functions, this year a completely unique capability was added to EIGER by employing a multi-layered media Green's function that can treat arbitrarily shaped objects that are penetrable. These extensions allow applications to be addressed in a variety of areas such as multi-layered electronic circuit boards, highpowered optical mirrors, and geophysical problems. 
Extensions to the Green's functions in the code are presently underway. General symmetry treatments have been added to the analysis code and are being incorporated into the post processor. Also, an effort to incorporate general periodic analysis, that was previously developed, for treatment of cavities and array analysis has also been completed. Recently, an effort was started to include Fast Multi-pole Methods (FMM) into EIGER.

\section{Organization and Usage}

EIGER presently consists of three distinct phases: a pre-processor, the physics solver, and a post-processor. The combined versatility of elements, basis functions, etc., suggests multiple combinations and approaches to solve models, possibly overwhelming the modeler with choices. However, this versatility also provides the flexibility to tackle portions of a model with the most appropriate combinations. Efforts are continuing to develop and extend the pre-processor to aid in the efficient usage of the tools. Postprocessing capabilities include the calculation of near and far fields from the basic current solutions, in addition to antenna gain, input impedance, and RCS.

A user of EIGER has a choice of basic numerical solutions of linear systems by either direct (serial and parallel) or iterative (serial) solvers. Additional work is in progress to create advanced linear solvers for high performance computer platforms.

\section{Examples}

Figure 1 shows the induced surface currents on an ALCM due to a plane wave excitation. The frequency of operation is $1 \mathrm{GHz}$. The compute mesh contained about 82,000 unknowns.

\section{Acknowledgments}

This work was performed under the auspices of the US Department of Energy by Lawrence Livermore National Laboratory under contract No. W-7405-Eng-48.

\section{References}

1. S. V. Yesantharao, "EMPACK-A software toolbox of potential integrals for computational electromagnetics," M.S.E.E. Thesis, University of Houston, Dec. 1989.

2. W. A. Johnson, D. R. Wilton, and R. M. Sharpe, "Modeling scattering from and radiation by arbitrary shaped objects with the Electric Field Integral Equation Surface Patch Code," Electromagnetics 10:41-63, 1990.

3. R. Graglia, D. Wilton, R. Peterson, "Higher order interpolatory vector bases for computational electromagnetics," IEEE Trans. Ant. Prop., V(45), N(3), pp. 329-342, 1997. 
Figures

Figure 1. Surface currents on an ALCM.

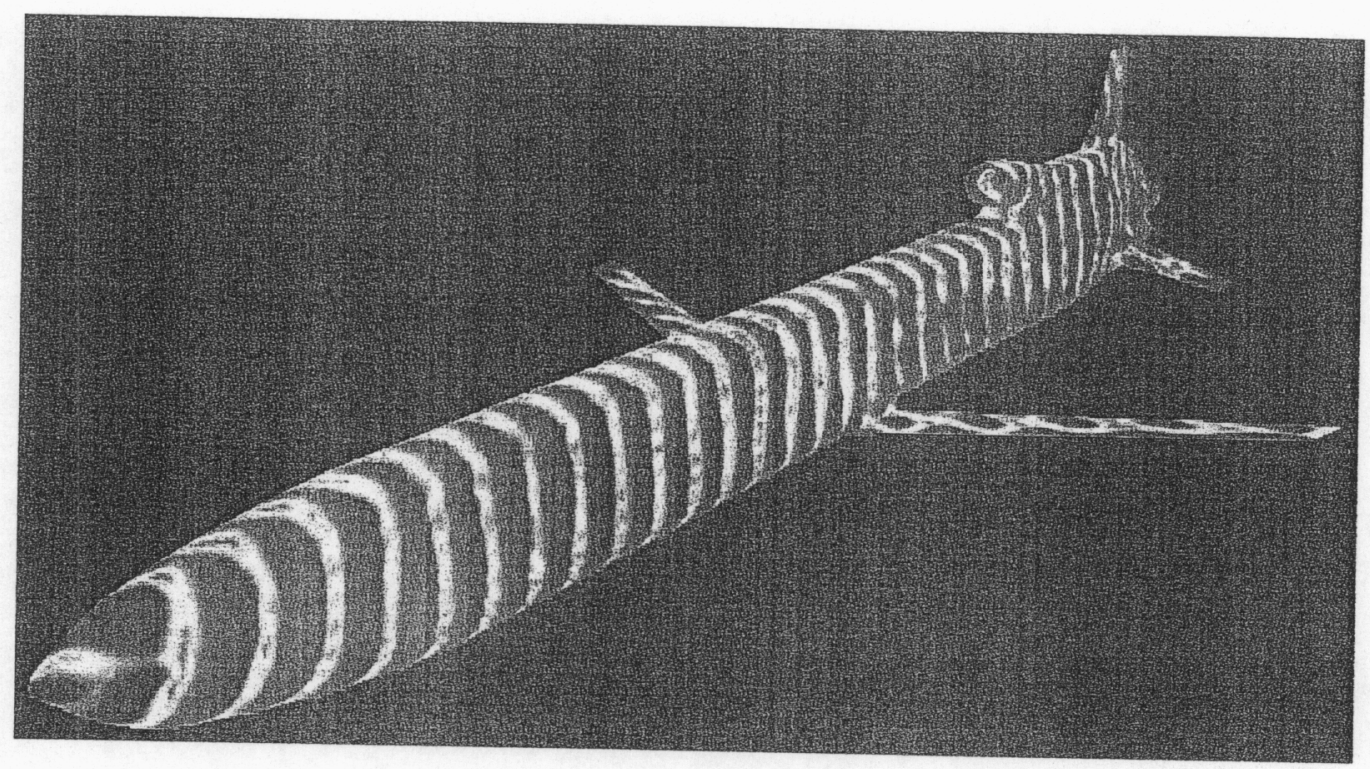

